\author{
Harriet C. Thoeny \\ Urs E. Studer \\ Stephan Madersbacher \\ Martin J. Sonnenschein \\ Peter Vock
}

\section{Caveats when interpreting intravenous urograms following ileal orthotopic bladder substitution}

Received: 16 June 2003

Revised: 27 October 2003

Accepted: 7 December 2003

Published online: 17 February 2004

(C) Springer-Verlag 2004

H. C. Thoeny $(\bullet) \cdot$ M. J. Sonnenschein

P. Vock

Department of Diagnostic Radiology,

University Hospital of Bern,

3010 Bern, Switzerland

e-mail: harriet.thoeny@insel.ch

Tel.: +41-31-6323641

Fax: +41-31-6322180

U. E. Studer $\cdot$ S. Madersbacher

Department of Urology,

University Hospital of Bern,

3010 Bern, Switzerland

\begin{abstract}
The aim of this study was to evaluate the appearance of the upper urinary tract following cystectomy and ileal orthotopic bladder substitution. Intravenous urograms (IVUs) performed preoperatively and at regular intervals postoperatively on 87 long-term survivors (minimum survival, 5 years) following ileal orthotopic bladder substitution were reviewed. Distention of the collecting system with blunted or rounded fornices was defined as dilatation. If in addition contrast medium excretion was delayed on the 5 -min film, this was defined as obstruction. Collecting system dilatation was present on all IVU films obtained from most patients $(80 \%)$ within 6 months of surgery, even in the absence of urinary tract obstruction. In contrast, dilatation was com-
\end{abstract}

monly seen only on the 20-min postinjection films (79\%) on urograms performed more than 1 year following surgery, but not encountered on the other two postinjection radiographs (at 5 and $60 \mathrm{~min}$ ). Five years after surgery, permanent obstruction was observed in only five $(3 \%)$ renoureteral units. Dilatation of the upper urinary tract after ileal orthotopic bladder substitution is a frequent finding on the 5-min, 20-min, and 60-min films during the early postoperative period but is found only on the 20-min film 1 year and later after surgery. These findings should not be overinterpreted as obstruction.

Keywords Urinary tract · Kidney · Bladder · Surgery $\cdot$ Urography

\section{Introduction}

In the past two decades orthotopic bladder substitution has become the preferred method of urinary diversion, particularly after radical cystectomy for invasive transitional cell cancer (TCC) of the urinary bladder [1-4]. Currently, the most popular methods use a segment of the terminal ileum which is cross-folded to form the bladder substitute and subsequently anastomosed to the urethral stump [1-4]. At our institution a dynamic antireflux mechanism by an isoperistaltic afferent tubular segment has been used in more than 400 patients since 1984 [4-6].

Patients undergoing orthotopic bladder substitution are regularly monitored by intravenous urographic (IVU) studies, primarily to exclude recurrent TCC of the upper urinary tract. It is therefore important for radiologists to be aware of specific findings on IVUs after ileal bladder substitution with an afferent tubular segment [7].

\footnotetext{
Patients and methods

Patients

Of the patients who received an ileal low-pressure orthotopic bladder substitute with an afferent tubular ileal segment as a dynamic antireflux mechanism at our institution between April 1986 and June 1997, 98 patients (96 men, 2 women) survived 5 years or longer. Indications for surgery were bladder cancer in the 96 men (without preoperative irradiation) and postradiogenic shrinkage of
} 


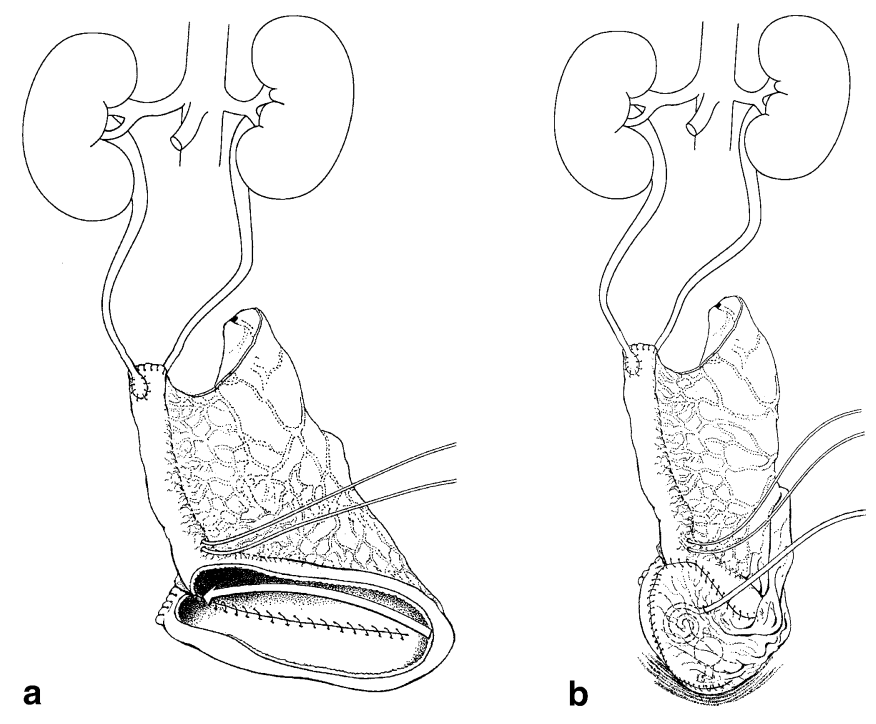

Fig. 1a,b Ileal low-pressure bladder substitute. a Ureters are anastomosed to the afferent tubular ileal segment without an antireflux mechanism. Oversewing of the two distal antimesenteric borders of the opened ileum results in a "U", which is subsequently crossfolded to form the reservoir. b Final appearance of the low-pressure reservoir which is anastomosed to the membranous urethra

the bladder in the 2 female patients. Eleven patients (11\%) were excluded: three were lost to follow-up and the remaining eight had incomplete radiological follow-up by IVU (in these patients CT or sonography was performed instead). The remaining 87 patients formed the basis of this study.

\section{Surgical technique}

After cystectomy, a 54-60-cm-long ileal segment was isolated approximately $25 \mathrm{~cm}$ proximal to the ileocecal valve $[4,8,9]$. The proximal part of the isolated segment $(14-16 \mathrm{~cm})$ formed the afferent tubular isoperistaltic ileal segment to which the ureters were anastomosed in an open end-to-side fashion (Fig. 1a). For the construction of the reservoir, the distal 40-44-cm segment was crossfolded twice to obtain a spherical low-pressure reservoir. The most dependent part of the constructed reservoir was then anastomosed to the membranous urethra (Fig. 1b) $[6,9,10]$.

\section{Intravenous urography}

All 87 patients underwent intravenous urography (IVU) preoperatively as well as in the late postoperative period, i.e., 1 year and later after surgery at yearly intervals. Only a subgroup of 40 patients operated on before 1994 had an IVU in addition during the early postoperative period (i.e., 1-6 months after surgery). These early examinations were abandoned later due to lack of clinical consequences.

After a KUB was obtained, $100 \mathrm{ml}$ of nonionic contrast material with an iodine concentration of $370 \mathrm{mg} / \mathrm{ml}$ (Iopamidol, Iopamiro 370; Bracco, Milan, Italy) was injected intravenously (time of injection, approx. $60 \mathrm{~s}$ ) by the supervising radiologist. Nontomographic views of the abdomen and pelvis (anteroposterior radiographs) were obtained 5 and 20 min after contrast medium administration. After the 20-min film compression, tomography of the kidneys was performed to exclude recurrent TCC of the upper urinary tract. The examination was completed with a postvoid film

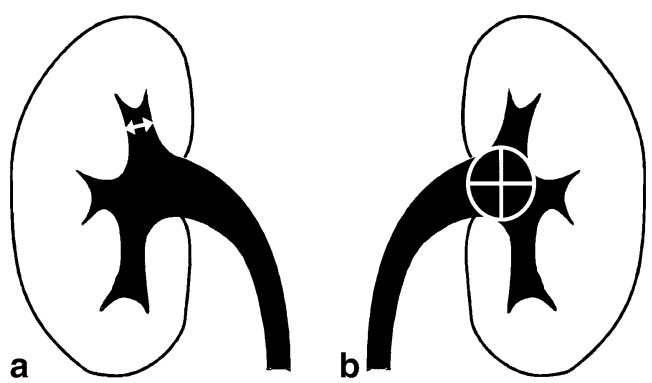

Fig. 2a,b Changes in the upper urinary tract were assessed by comparing the following parameters: a Calyceal neck width was measured in millimeters at the same level in all films. b Renal pelvis area was measured in centimeters squared as length $\times$ width in a circle projected into the renal pelvis
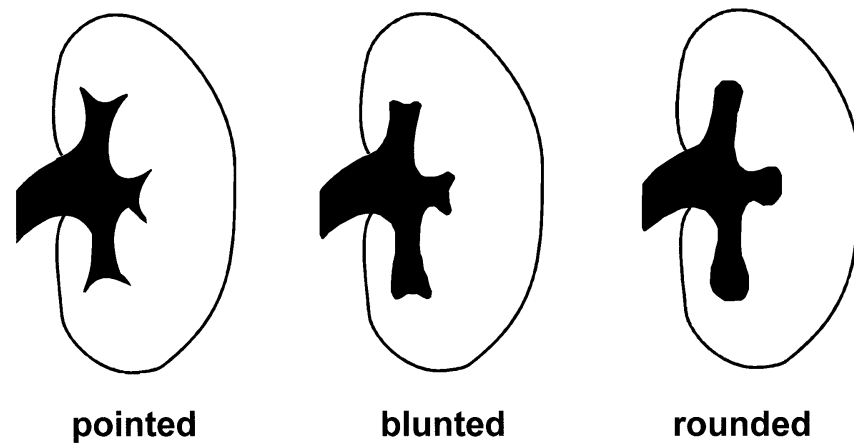

Fig. 3 Morphologic classification of the pelvicalyceal system. Appearance of the fornices classified as one of three types: point$e d$, blunted, and rounded. The presence of rounded or blunted fornices and no delay in contrast medium excretion was defined as dilatation

in the upright position 60 min after injection. Contrast medium excretion on the 5-min film as well as complete drainage of the collecting system on a postvoid film after 60 min were considered as evidence that no significant obstruction was present.

The following changes were analyzed on the 5-min, 20-min, and 60-min films:

1. Width of calyceal neck: this was measured in millimeters at the level where an optimal measurement could be obtained, and always at the same level on all films of the same day (Fig. 2a).

2. The renal pelvis area was measured in centimeters squared as length $\times$ width in a circle projected into the renal pelvis (Fig. 2b)

3. The appearance of the pelvicalyceal system was classified as one of three types (pointed, blunted, rounded) according to the shape of the fornices (Fig. 3).

Statistical analysis was carried out with Microsoft Excel. Numerical data are reported as means \pm standard deviation (SD).

For this study a total of 733 IVUs were reviewed independently by two radiologists (preoperatively, $n=87$ IVUs; at 1 month, $n=36$; at 6 months, $n=26$; 1 year, $n=87 ; 2$ years, $n=87 ; 3$ years, $n=87 ; 4$ years, $n=87 ; 5$ years, $n=87 ; 6$ years, $n=54 ; 7$ years, $n=41$; 8 years, $n=26$; 9 years, $n=11 ; 10$ years $n=8 ; 11$ years, $n=5$; 12 years, $n=3 ; 13$ years, $n=1)$. In case of disagreement a consensus opinion was reached. 


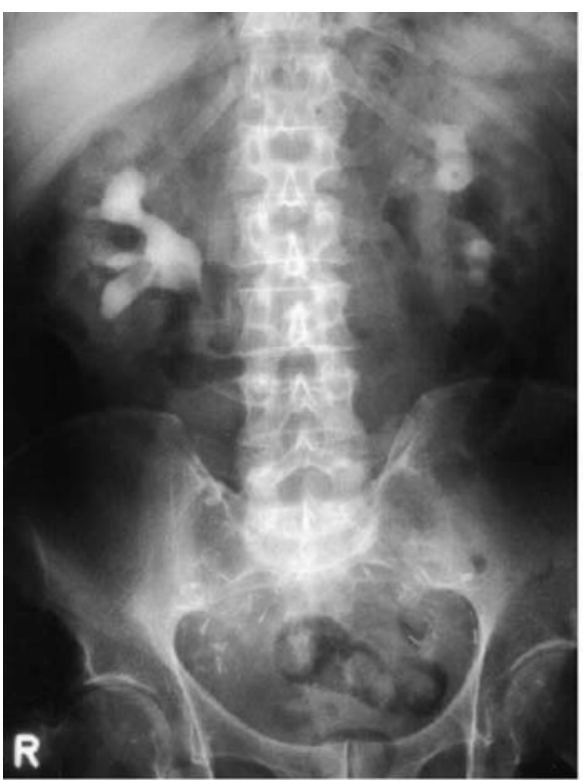

\section{$5 \mathrm{~min}$}

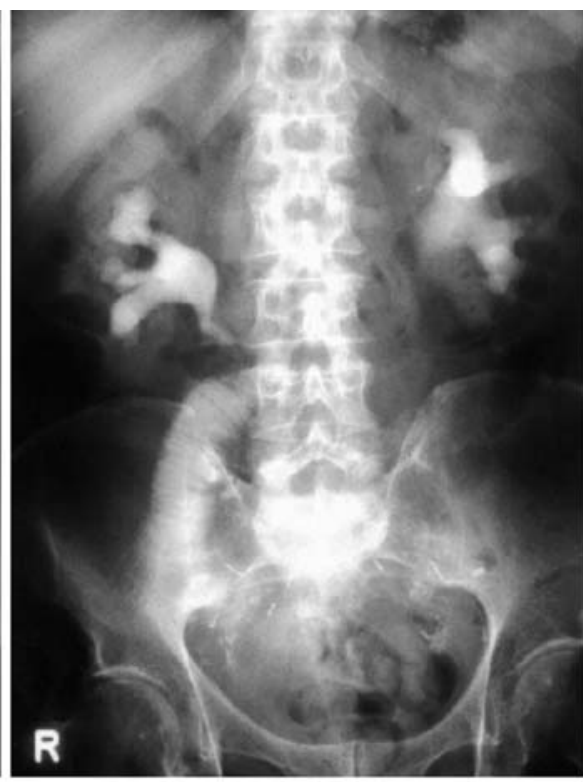

$20 \mathrm{~min}$

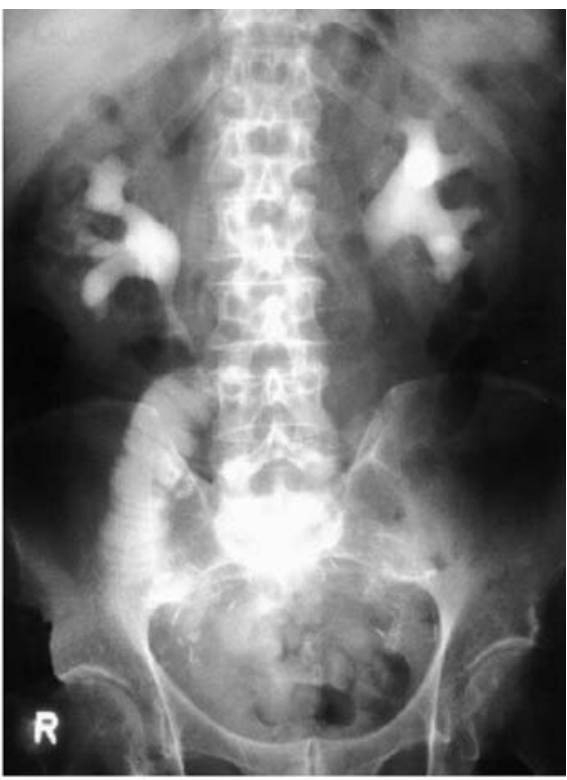

$60 \mathrm{~min}$
Fig. 4 Radiographic appearance of the upper urinary tract in the early postoperative period. A typical finding in the early postoperative period (1-6 months postop) is a persistent dilatation of the upper uri- nary tract with blunted or rounded fornices and distention of the whole collecting system on the 5-min, 20-min, and 60-min films.

Note that contrast medium excretion is on time on the 5-min film

Table 1 Changes of the upper urinary tract in the early (1-6 months after surgery) and late (at 60 months) postoperative period (postop)

\begin{tabular}{llllll}
\hline Postinjection time & \multicolumn{2}{l}{ Calyceal neck width $(\mathrm{mm})$} & & \multicolumn{2}{l}{ Renal pelvis area $\left(\mathrm{cm}^{2}\right)$} \\
\cline { 2 - 3 } & Early postop $(n=80)$ & Late postop $(n=170)$ & & Early postop $(n=80)$ & Late postop $(n=170)$ \\
\hline $5 \mathrm{~min}$ & $6.6 \pm 2.5$ & $5.5 \pm 3.0$ & $6.5 \pm 3.7$ & $5.8 \pm 2.6$ \\
$20 \mathrm{~min}$ & $6.8 \pm 3.6$ & $8.3 \pm 6.4$ & $6.7 \pm 4.6$ & $8.4 \pm 5.4$ \\
$60 \mathrm{~min}$ & $6.0 \pm 3.2$ & $4.8 \pm 3.3$ & $6.2 \pm 2.8$ & $4.9 \pm 5.1$ \\
\hline
\end{tabular}

\section{Results}

Patients

The median age of the 87 patients was 62 years (range, $36-80$ years) at the time of surgery and the median postoperative observation period was 80 months (range, 60-155 months). Preoperative IVUs were available for all patients (174 renoureteral units).

One year and later after surgery, regular IVU followup at yearly intervals was available for all patients over a minimum observation time of 5 years.

During follow-up four patients underwent unilateral nephroureterectomy for recurrent TCC of the upper urinary tract diagnosed by performing IVU after a median of 46 months (range: $28-62$ months) following cystectomy. This left 170 renoureteral units for regular long-term ( $\geq 5$ years) IVU analysis.
Dilatation of the upper urinary tract

Preoperatively, $152(87.4 \%)$ of 174 renoureteral units were normal with no obstruction and no dilatation (pointed fornices), 16 (9.2\%) were dilated (blunted or rounded fornices) without delayed contrast medium excretion, and $6(3.4 \%)$ were obstructed.

In the early postoperative period (1-6 months postoperatively), $64(80 \%)$ of 80 renoureteral units were dilated on the 5-min, 20-min, and 60-min films (Table 1, Fig. 4). A dilatation limited to the 20-min film with pointed fornices in the 5-min and 60-min films was observed in $6(7.5 \%)$ of 80 renoureteral units. In these six units an additional significant increase in calyceal neck width and renal pelvis area from the 5-min to the 20min film and a decrease in size of these parameters from the 20-min to the 60-min film were observed. Ten $(12.5 \%)$ of the 80 renoureteral units showed no dilata- 


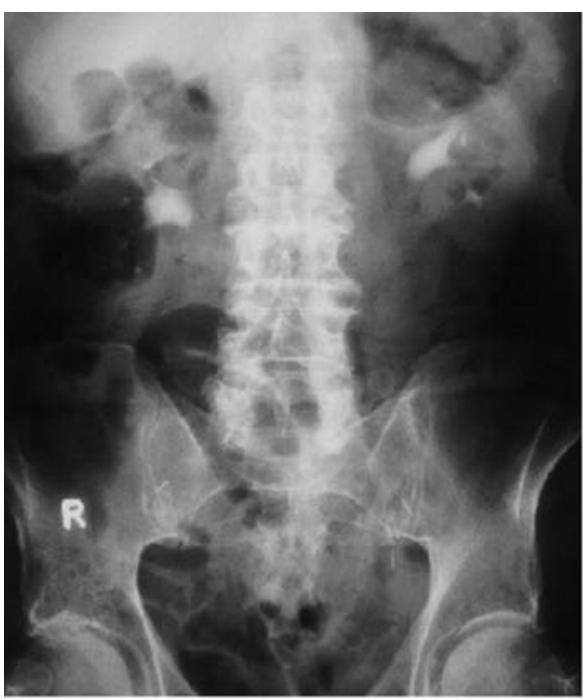

$5 \mathrm{~min}$

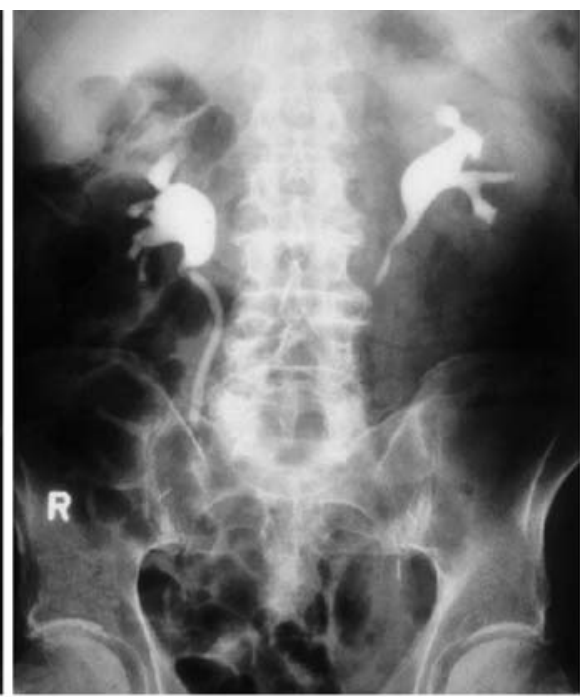

$20 \mathrm{~min}$

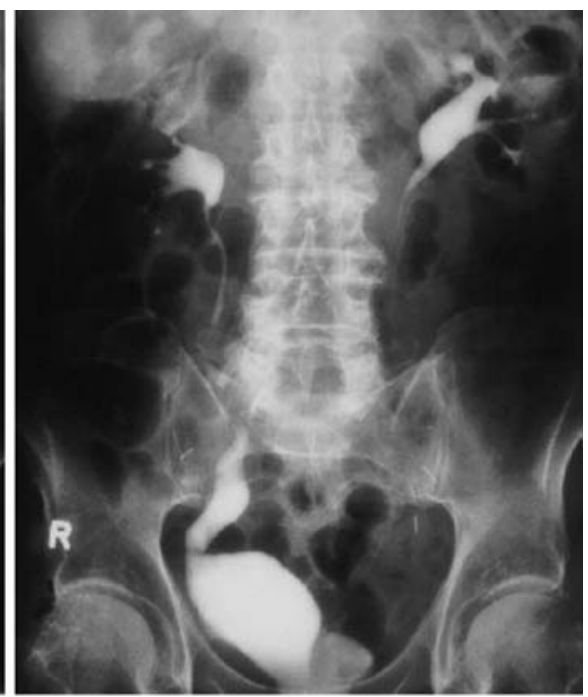

$60 \mathrm{~min}$
Fig. 5 Radiographic appearance of the upper urinary tract in the late postoperative period. In the late postoperative period (here 10 years after surgery) dilatation of the upper urinary tract is limited to the 20-min film. The upper urinary tract is normal on the 5-min and 60 -min films with pointed fornices

tion with pointed fornices on the 5-min, 20-min, and 60-min films.

In the late postoperative period, however, 135 (79\%) of 170 renoureteral units had normal-appearing upper urinary tracts with pointed fornices on the 5-min and 60min films, but a dilatation (blunted/rounded fornices) only on the 20-min film. Concomitantly, calyceal neck width and renal pelvis area increased from the 5-min to the 20-min film and then decreased from the 20-min to the 60 -min film (Table 1, Fig. 5). Fourteen (8\%) of 170 renoureteral units were dilated throughout the entire investigation. Seven of these 14 units were already dilated preoperatively and seven were normal before surgery. No dilatation throughout the entire investigation was observed in $16(9 \%)$ of the 170 renoureteral units.

In the 16 renoureteral units with preoperative dilatation, this persisted ( $\geq 5$ years) in seven and became normal in eight cases. One patient underwent nephroureterectomy 5 years after surgery due to recurrent TCC of the upper urinary tract.

\section{Obstruction}

In preoperative IVUs, obstruction (i.e., delayed contrast medium excretion) was present in $6(3 \%)$ of the 174 renoureteral units due to tumor infiltration $(n=5)$ or ureterolithiasis $(n=1)$. After follow-up of 5 years or more, obstruction persisted in three of these units and in two obstruction resolved. One patient underwent unilateral nephroureterectomy of the preoperatively obstructed kidney 5 years after surgery for recurrent TCC.

A de novo and persistent obstruction due to a ureteroileal anastomotic stricture occurred in two (1.1\%) renoureteral units 6 months and 1 year after surgery.

A transient obstruction due to ureteroileal anastomotic stricture occurred in three $(2 \%)$ of the renoureteral units in two patients (6-60 months after surgery) with complete reversal following endourological intervention.

In the long-term (5-13 years after surgery), no obstruction of the upper urinary tract was observed in the vast majority of the renoureteral units $(164 / 170=96 \%)$.

\section{Discussion}

This is the first detailed report on specific radiographic alterations of the upper urinary tract after orthotopic bladder substitution with an afferent tubular segment evaluating early (1-6 months after surgery) and late ( $\geq 5$ years) postoperative findings [7]. IVUs in the early postoperative phase were only available in a subset of patients $(n=40)$ for two reasons. Firstly, because IVU studies in the early postoperative phase show dilatation of the upper urinary tract in the vast majority of cases; this is clinically irrelevant because it resolves spontaneously with further follow-up. Similar observations have been made with other forms of urinary diversions [10-12]. Secondly, IVUs in the early phase are not necessary to exclude upper urinary tract recurrences because these develop later (28-64 months after surgery in our series). Therefore, we removed IVU 1-6 months after surgery from our follow-up protocol in the early 1990s and replaced it by ultrasonography. 
The high incidence of dilatation $(80 \%)$ of the upper urinary tract on the 5-min, 20-min, and 60-min films within the first 6 months after surgery may suggest obstruction. However, we only found two cases of mechanical obstruction caused by unilateral ureteroileal stenosis with subsequent hydronephrosis. The most plausible explanation for the constant dilatation on the 5-min, 20-min, and 60-min films in the early postoperative period is lack of ureteric peristalsis which is additionally maintained by the frequently elevated pressure in the initially small-capacity reservoirs. The higher the end-fill pressure in the reservoir, the higher the pressure in the renal pelvis. Simultaneous intrapyelic (by a nephrostomy tube), intravesical, and intraabdominal urodynamic studies clearly excluded reflux as a potential reason for upper tract dilatation in patients with an afferent tubular segment [13]. Irrespective of the exact mechanism leading to dilatation of the upper urinary tract during the early postoperative period in patients with various types of bladder substitutes, it is important for radiologists to know that it exists and that it is a frequent urographic finding which should not be overinterpreted as obstruction.

One year and later after surgery, dilatation of the upper urinary tract under physiological conditions was limited to the 20-min film with normal findings at 5 and $60 \mathrm{~min}$ in our series. Obviously, reasons other than those responsible for the persistent dilatation in the early postoperative period must cause this transient dilatation [14]. It cannot be an elevated reservoir pressure, as 1 year after surgery the reservoir is a low-pressure one with a capacity around $450 \mathrm{ml}[4,15]$. In contrast to the true bladder, orthotopic intestinal low-pressure reservoirs have no coordinated contraction during voiding. Thus, an isolated intravesical pressure rise, which could cause urine reflux and an increase in intrapyelic pressure, is not possible. Moreover, the patients who received an ileal lowpressure bladder substitute with an antireflux flap valve showed the same transient dilatation on the $20 \mathrm{~min}$ film as did the patients with an afferent tubular ileal segment without a flap valve mechanism [12, 13, 16]. Finally, spontaneous normalization of the upper urinary tract morphology with dilatation limited to the 20 -min postcontrast film and a normal-appearing collecting system on the 5-min and 60-min films 1 year and later after surgery would not be expected if reflux were the cause, because the changes would be constant. Although the degree of dilatation of the upper urinary tract on an IVU can to some extent be related to the state of hydration, diuresis, renal function, or pathologic obstruction, we believe that the transient dilatation seen only on the 20min film in the late postoperative period is caused by an unphysiologically high contrast-medium-induced diuresis which exceeds the transport capacity of the afferent tubular segment $[6,17]$. Thus, during maximal diuresis the limited transport capacity of the interposed ileal segment may lead to transient functional obstruction.

It is of major clinical relevance to discriminate the described temporary functional dilatation of the upper urinary tract after 20 min from one caused by a mechanical outlet obstruction, which is most often due to a ureteric stenosis. The latter would require prompt urologic intervention in order to prevent irreversible kidney damage. The discrimination between dilatation only and obstruction is best made by judging whether contrast medium excretion is present or delayed on the 5-min film and whether the collecting system is drained after $60 \mathrm{~min}$. Misinterpretation of the transient dilatation as true obstruction would lead to unnecessary additional studies (e.g., scintigraphy studies) or interventions (e.g., percutaneous nephrostomies).

MR urography is an interesting and valuable alternative for the detection and classification of obstruction after orthotopic urinary diversion, particularly in patients with impaired renal function $[18,19]$. However, it does not allow detection of smaller urothelial tumors in a substantial number of patients [20]. This issue may be of particular importance in the follow-up of patients operated for bladder cancer.

In conclusion, after orthotopic ileal bladder substitution, dilatation of the upper urinary tract 5, 20, and 60 min after contrast medium injection is a very frequent (80\%) finding in the early postoperative period. It is of major clinical relevance not to overinterpret this functional dilatation as being the result of a mechanical outlet obstruction. This is only likely if contrast medium excretion is delayed. One year and later after surgery, dilatation of the renal collecting system should be limited to the 20-min film, i.e., the phase of maximal contrast-medium-induced diuresis. To avoid misdiagnosis of obstruction, radiologists and urologists must be aware of these specific urographic findings of the upper urinary tract after ileal bladder substitution with an afferent tubular segment in the early and late postoperative period. Discrimination can be made based on the aspect of the 5-min and, eventually, the 60-min film.

\section{References}

1. Burkhard FC, Studer UE (2000) Orthotopic bladder substitution. Curr Opin Urol 10:343-349
2. Elmajian DA, Stein JP, Skinner DG (1996) Orthotopic urinary diversion: the Kock ileal neobladder. World J Urol 14:40-46
3. Hautmann RE, de Petriconi R, Gottfried HW, Kleinschmidt K, Mattes R, Paiss T (1999) The ileal neobladder: complications and functional results in 363 patients after 11 years of followup. J Urol 161:422-427 
4. Studer UE, Zingg EJ (1997) Ileal orthotopic bladder substitutes. What we have learned from 12 years' experience with 200 patients? Urol Clin North Am 24:781-793

5. Studer UE, Danuser H, Merz VW, Springer JP, Zingg EJ (1995) Experience in 100 patients with an ileal lowpressure bladder substitute combined with an afferent tubular ileal segment. J Urol 154:49-56

6. Thoeny HC, Sonnenschein MJ, Madersbacher S, Vock P, Studer UE (2002) Is ileal orthotopic bladder substitution with an afferent tubular segment detrimental to the upper urinary tract in the long term? J Urol 168:2030-2034

7. Heaney MD, Francis IR, Cohan RH, Ellis JH, Tekchandani A, Montis JE, Korobkin M (1999) Orthotopic neobladder reconstruction: findings on excretory urography and CT. AJR 172:1213-1220

8. Burkhard FC, Studer UE (2001) Orthotopic urinary diversion using an ileal low-pressure bladder substitute with an afferent tubular segment. Atlas Urol Clin North Am 9:57-73
9. Danuser H, Studer UE (1998) Orthotopic urinary diversion using an ileal low-pressure reservoir with an afferent tubular segment. In: Graham SD Jr (ed) Glenn's urologic surgery, 5th edn. Lippincott-Raven Publishers, Philadelphia, pp 655-661

10. Abd-el-Gawad G, Abrahamsson K, Hanson E et al (1999) Kock urinary reservoir maturation in children and adolescents: consequences for kidney and upper urinary tract. Eur Urol 36:443-449

11. Chen KK, Chang LS, Chen MT, Yeh SH (1991) Prospective radionuclid renal function evaluation and its correlation with radiological findings in patients with Kock pouch urinary diversion. J Urol 145:952-955

12. Ralls PW, Barkos JA, Skinner DG, Boswell WD Jr, Radin DR, Colletti PM, Halls JM (1986) Imaging of the Kock continent ileal urinary reservoir. Radiology 161:477-483

13. Studer UE, Spiegel T, Casanova G, Springer J, Gerber E, Ackermann DK, Gurtner F, Zingg EJ (1991) Ileal bladder substitute: antireflux nipple or afferent tubular segment? Eur Urol 20:315-326

14. Lugagne PM, Hervé JM, Lebret T, Barre P, Mollier S, Botto H (1997) Ureteroileal implantation in orthotopic neobladder with the Le Duc-Camey mucosal-through technique: risk of stenosis and long-term followup. J Urol 158:765-767

15. Madersbacher S, Möhrle K, Burkhard F, Studer UE (2002) Long-term voiding pattern of patients with ileal orthotopic bladder substitutes. J Urol 167:2052-2057
16. Studer UE, Danuser H, Thalmann GN, Springer JP, Turner WH (1996) Antireflux nipples or afferent tubular segments in 70 patients with ileal low pressure bladder substitutes: long-term results of a prospective randomized trial. J Urol 156:1913-1917

17. Talner LB (1990) Obstructive uropathy. In: Pollack HM (ed) Clinical urography. WB Saunders Company, Philadelphia, pp 1535-1620

18. Borthne AS, Pierre-Jerome C, Gjesdal KI, Storaas T, Courivaud F, Eriksen M (2003) Pediatric excretory MR urography: comparative study of enhanced and non-enhanced techniques. Eur Radiol 13:1423-1427

19. Zielonko J, Studniarek M, Markuszewski M (2003) MR urography of obstructive uropathy: diagnostic value of the method in selected clinical groups. Eur Radiol 13:802-809

20. Bakker J, De Kort GA, Lo R, Van Gils AP, Hene RJ, Lock TM, Burger H, Beek FJ (2003) MR urography for the preoperative evaluation of living renal donors. Eur Radiol 12:2021-2029 